\title{
Insulin secreted from genetically engineered intestinal cells reduces blood glucose levels in diabetic mice
}

\begin{abstract}
Poorly controlled diabetes mellitus can result in serious complications. Gene therapy is increasingly being considered as an alternative approach to treat diabetes, because of its ability to induce physiological insulin secretion and it allows patients to escape insulin injections. The properties of gut $\mathrm{K}$ and L-cells, including glucose sensitivity, the ability to process insulin and a regulated secretion pathway support their use as surrogate $\beta$-cells. Previous in vitro studies have provided sufficient evidence supporting the use of these cells for gene therapy studies. Therefore, we examined the ability of $\mathrm{K}$ and L-cells to produce insulin in diabetic mice. Chitosan nanoparticles were used to transfer the insulin gene into intestinal cells via oral administration. The efficiency of chitosan as a gene vehicle was investigated through the use of reporter gene. Insulin mRNA and protein expression levels were measured by RT-PCR and ELISA, respectively. Blood glucose testing revealed that this treatment reduced glucose levels in diabetic mice. The decrease in blood glucose level in the first week of treatment was greater in mice with K-cell specific insulin expression compared with mice with L-cellspecific insulin expression. These results indicate that inducing insulin secretion in K-cells conferred a quicker response to gene therapy.
\end{abstract}

Keyword: Chitosan nanoparticles; Diabetes gene therapy; In vivo; K cells; L-cells 